\title{
Studies on crystallization of peptidyl-prolyl cis-trans isomerase and AreB from Aspergillus flavus
}

\begin{abstract}
Aspergillus flavus is a disease causative agent of many agriculture plants, common hosts are cereal grains, legumes, and tree nuts. Many strains produce a significant quantity of toxins named as mycotoxins. A. flavus is also an opportunistic pathogen of human and animals, that cause aspergillosis in immunocompromised individuals. A. flavus has Peptidylprolyl cis-trans isomerases (PPIase), which catalyze the isomerization of peptide bonds preceding proline residues, a process of high valuable for correct folding. PPIase belongs to the superfamily of a protein named as cyclophilin, which found in eukaryotic, bacterial and archaea. AreB hypothetical protein is similar to GATA transcription factors maintain transcription during growth and differentiation by identifying distinct GATA sites with a tandem of two conserved zinc fingers. Both zinc fingers wrap around a palindromic GATA site. This study will examine the protein structure from the X-ray crystals of PPIase and AreB and the structure determination of PPIase and AreB among the different species of A. flavus.
\end{abstract}

Keywords: aspergillus flavus, immunocompromised, peptidyl-prolyl cis-trans isomerases, cyclophilins, AreB, x-ray crystallization, GATA transcription factors

\author{
Volume 5 Issue I - 2017
}

\author{
Saleem Ahmad, ${ }^{1,2}$ Farman Ali ${ }^{2,3}$ \\ 'Key Laboratory of Pathogenic Fungi and Mycotoxins of Fujian \\ Province, Fujian Agriculture and Forestry University, China \\ ${ }^{2}$ Key Laboratory of Biopesticide and Chemical Biology of \\ Education Ministry and School of Life Science, Fujian Agriculture \\ and Forestry University, China \\ ${ }^{3}$ Key Laboratory of Agroecological Processing and Safety \\ Monitoring, Fujian Agriculture and Forestry University, China
}

\begin{abstract}
Correspondence: Saleem Ahmad, Key Laboratory of Biopesticide and Chemical Biology of Education Ministry, Key Laboratory of Pathogenic Fungi and Mycotoxins of Fujian Province and School of Life Science, Fujian Agriculture and Forestry University, Fuzhou 350002, China, Tel I8859|44980, Email saleem.chilas@yahoo.com
\end{abstract}

Received: June 25, 2017 | Published: July 05, 2017

\section{Introduction}

Peptidyl-prolyl cis-trans isomerases (PPIase) is also known as rotamase or cyclophilins, ${ }^{1}$ catalyze cis-trans isomerization of peptide bonds, preferring those preceding proline residues. ${ }^{2}$ The enzyme can be found in a large variety of organisms such as bacteria, plants, and mammals, sometimes as single domain proteins, but also as components in larger complexes. ${ }^{3}$ PPIase accelerate protein folding both in vivo and in-vitro. They bind to and mediate the biological effects of the immunosuppressive agent cyclosporine $\mathrm{A},{ }^{4}$ they participate in cell surface recognition ${ }^{5}$ and heat-shock response. Cyclophilins are a diverse family regarding function and have incriminated in protein folding processes which rely on catalytic/ chaperone-like activities. ${ }^{7}$ Biological processes that reliable on the action of proteins and their domains, for example, folding collaborator proteins assist protein folding as disulfide isomerases or peptidylprolyl cis/trans isomerases. ${ }^{8}$ These proteins have evolved to recognize precise signatures of protein sequences and manage in vivo protein folding. ${ }^{9}$ A considerable number of proteins have acknowledged holding peptidyl-prolyl cis/trans isomerase a domain that has been constituting another specific protein recognition unit. ${ }^{10}$ The peptidylprolyl cis/trans isomerases are enzymes that gather speed the slow cis/ trans isomerization of peptidyl-prolyl bonds in different folding states of an intention protein. PPIase-catalysed protein conformational changes happened during the refolding of denatured proteins, ${ }^{11} \mathrm{de}$ novo protein synthesis and the arrangement of biologically active conformations of polypeptides, ${ }^{12}$ Based on drug specificity and primary sequence homology. PPIases have been separated into three distinct families: $:^{13}$ a) the cyclosporin A (CsA)-binding proteins, cyclophilins,) the FK506 and rapamycin binding proteins, FKBPs,). The parvulins, do not bind immunosuppressant drugs..$^{14}$ Even though cyclophilins and FKBPs were identified for some decades, the cellular purpose of these enzymes is not yet absolutely understood. They concerned in the folding of newly synthesized proteins, transport and gathering of fundamental cellular protein complexes, ${ }^{15}$ In contrast, the purpose of a part of the third PPIase family, Pin 1 could expose in much more details, and a significant task in the cell cycle mechanism in eukaryotes was proved. ${ }^{16}$

The GATA-binding proteins are a collection of structurally associated transcription factors that bind to the DNA compromise sequence GATA. ${ }^{17}$ Members of the GATA protein family (GATA16) purpose as lineage-specific transcription factors for a digit of cell types in the hematopoietic system. ${ }^{18}$ For example, GATA1 is basic for erythroid and megakaryocytic development. ${ }^{18}$ GATA2 plays a key role in regulating the transcription of genes involved in the improvement and proliferation of hematopoietic ${ }^{19}$ and the endocrine cell lineages GATA3 is a significant regulator of $\mathrm{T}$ cell improvement, including $\mathrm{Th} 2,{ }^{20}$ and regulatory $\mathrm{T}$ cells,${ }^{21}$ and plays a major role in endothelial cell biology and GATA4 regulates genes occupied in embryogenesis, and myocardial separation and function. ${ }^{22}$ The function of GATA proteins depends critically on two highly conserved zinc fingers and nearby basic regions. ${ }^{23}$ The C-terminal "C-finger" and its adjacent basic region is necessary and sufficient for GATA to bind its cognate sequence, WGATAR (W=A/T, R=A/G. ${ }^{24}$ The $\mathrm{N}$-terminal "N-finger" also bind DNA independently but has a preference for GATC core motifs. ${ }^{25}$ Both fingers participate in binding the palindromic GATA motif ATCWGATA $(\mathrm{W}=\mathrm{A} / \mathrm{T})$, resulting in markedly increased affinity, ${ }^{26}$ GATA proteins give to transcriptional regulation by facilitating chromosome looping, thereby mediating long-range manage of gene appearance in the nucleus. For example, GATA1 and its cofactor FOG-1 openly engage looped enhancers and mark gene promoters at the b-globin locus. ${ }^{27}$ However, the molecular mechanism by which GATA and Friend of GATA (FOG) proteins mediate loop formation remains unclear. Structural studies based on both nuclear magnetic resonances (NMR) spectroscopy and X-ray crystallography ${ }^{28}$ has characterized the DNA-binding mechanisms of the GATA C-finger. ${ }^{29}$ In addition, the structure of the GATA1 N-finger bound to the FOG-1 zinc finger 1 has been distinguished by NMR. ${ }^{30}$ 


\section{Crystallization}

For a protein to crystallize, it must reach the thermodynamically unstable state of supersaturation. ${ }^{31}$ Crystallization experiments are carried out by the sitting-drop method at $16^{\circ} \mathrm{C}$ using 96 -well plates, common precipitants are salts, like ammonium sulfate, organic solvents, including ethylene glycol and polyethylene glycol (PEG) 200 , or different polymers, represented mostly by larger PEGs. The commercial crystallization kits Index, PEG/Ion, PEG/Ion2, Crystal Screen1, Crystal Screen 2 and Natrix is used to find the initial conditions for crystal growth. There are certain parameters affecting crystallization like temperature, $\mathrm{pH}$ and protein concentration. ${ }^{32}$

\section{X-ray crystallography, data collection \& data processing}

When accelerated electrons hit metal, energy is released in the form of X-ray photons. ${ }^{33}$ The X-rays can be looked upon as particles, ${ }^{34}$ but also as waves having a specific amplitude and phase. During data collection, the crystal exposed to X-rays of a specific wavelength, ${ }^{35}$ ranging from $0.5-1.6 \AA$ for a typical experiment. When the X-rays hit the crystal, most of them are straight through, but some are hit the atoms in the crystal, causing them to oscillate. The X-rays is remitted with the same amplitude and phase as before but in a different angle. Before data collection, crystals are soaked in a cryo protectant solution. ${ }^{36}$

\section{Solve phase problem}

More than a few methods can be used to resolve the phase problem like single isomorphous replacement method (SIR), ${ }^{37}$ Multiple Isomorphous Replacement, ${ }^{37}$ (MIR), single wavelength anomalous dispersion (SAD), ${ }^{38}$ and multiple wavelengths anomalous dispersion (MAD), ${ }^{37}$ One can also combine the two techniques, use single or multiple isomorphous replacements together with anomalous dispersion, referred to as SIRAS and MIRAS, respectively. After crystallographic data collection, all data sets are indexed and integrated with MOSFLM. ${ }^{37}$

\section{Conclusion}

Analysis of the published data shows that PPIase and AreB hypothetical protein similar to GATA transcription factor from A. flavus are not expressed, purified and crystallized as well their structures are not determined. But their relative solved structures are found in Protein Data Bank (PDB) from different species. Crystallization is necessary to get the three-dimensional structure of nucleic acids and proteins; it often represents the bottleneck in structure determination. Our assimilation of crystallization mechanisms regarding PPIase and AreB is still incomplete. In this review, we emphasize essential feature of the crystallization process.

\section{Acknowledgements}

None.

\section{Conflict of interest}

The author declares no conflict of interest.

\section{References}

1. Liu J, Walsh CT. Peptidyl-prolyl cis-trans-isomerase from Escherichia coli: a periplasmic homolog of cyclophilin that is not inhibited by cyclosporin A. Proc Natl Acad Sci USA. 1990;87(11):4028-4032.
2. Deuerling E, Bukau B. Chaperone-assisted folding of newly synthesized proteins in the cytosol. Crit Rev Biochem Mol Biol. 2004;39(5-6):261277.

3. Martinez-Hackert E, Hendrickson WA. Structures of and interactions between domains of trigger factor from Thermotoga maritima. Acta Crystallogr D Biol Crystallogr. 2007;63(4):536-547.

4. Colgan J, Asmal M, Yu B, et al. Cyclophilin A-deficient mice are resistant to immunosuppression by cyclosporine. J Immunol. 2005;174(10):60306038.

5. Fang ZF, Gai H, Huang YZ, et al. Rabbit embryonic stem cell lines derived from fertilized, parthenogenetic or somatic cell nuclear transfer embryos. Exp Cell Res. 2006;312(18):3669-3682.

6. Lin J, Adams LG, Ficht TA. Characterization of the heat shock response in Brucella abortus and isolation of the genes encoding the GroE heat shock proteins. Infect Immun. 1992;60(6):2425-2431.

7. Yerbury JJ, Poon S, Meehan S, et al. The extracellular chaperone clusterin influences amyloid formation and toxicity by interacting with prefibrillar structures. FASEB J. 2007;21(10):2312-2322.

8. Van Damme M, Crompot E, Meuleman N, et al. Characterization of TET and IDH gene expression in chronic lymphocytic leukemia:comparison with normal B cells and prognostic significance. Clin Epigenetics. 2016;8(1):132.

9. Bairoch A. The PROSITE dictionary of sites and patterns in proteins, its current status. Nucleic Acids Res. 1993;21(13):3097.

10. Chen Y, Bates DL, Dey R, et al. DNA binding by GATA transcription factor suggests mechanisms of DNA looping and long-range gene regulation. Cell rep. 2012;2(5):1197-1206.

11. Crompton M. On the involvement of mitochondrial intermembrane junctional complexes in apoptosis. Curr Med Chem. 2003;10(16):14731484.

12. Zawadzki M. Smoking article and smoking article filter. Google Patents. 2003.

13. Ranganathan $\mathrm{R}$, Lu KP, Hunter $\mathrm{T}$, et al. Structural and functional analysis of the mitotic rotamase Pin1 suggests substrate recognition is phosphorylation dependent. Cell. 1997;89(6):875-886.

14. Hong OK, Suh SH, Kwon HS, et al. Proteomic analysis of differential protein expression in response to epidermal growth factor in neonatal porcine pancreatic cell monolayers. J Cell Biochem. 2005;95(4):769781.

15. Jäger S, Kim DY, Hultquist JF, et al. Vif hijacks CBF-[bgr] to degrade APOBEC3G and promote HIV-1 infection. Nature. 2012;481(7381):371-375.

16. Ryo A, Suizu F, Yoshida $\mathrm{Y}$, et al. Regulation of $\mathrm{NF}-\kappa \mathrm{B}$ signaling by Pin1-dependent prolyl isomerization and ubiquitin-mediated proteolysis of p65/RelA. Mol Cell. 2003;12(6):1413-1426.

17. Arceci RJ, King AA, Simon MC, et al. Mouse GATA-4: a retinoic acidinducible GATA-binding transcription factor expressed in endodermally derived tissues and heart. Mol Cell Bio. 1993;13(4):2235-2246.

18. Wang J, Guo LP, Chen LZ, et al. Identification of cancer stem cell-like side population cells in human nasopharyngeal carcinoma cell line. Cancer Res. 2007;67(8):3716-3724.

19. Georgescu C, William JRL, Deirdre DSA, et al. A gene regulatory network armature for T lymphocyte specification. Proceedings of the National Academy of Sciences. 2008;105(51):20100-20105.

20. Syed MA, Arshad Saleem Bhatti, Chenzhong Li, et al. Use of SPR biosensor for the study of proteolytic action of a serine protease enzyme. Am J Biomed Sci. 2011;3(4):253-257. 
21. Lindblad-Toh K, Wade CM, Mikkelsen TS, et al. Genome sequence, comparative analysis and haplotype structure of the domestic dog. Nature. 2005;438(7069):803-819.

22. Lin CR. Pitx 2 regulates lung asymmetry, cardiac positioning and pituitary and tooth morphogenesis. Nature. 1999;401(6750):279-282.

23. Li W, Zhang J, Su Y, et al. Effects of zinc binding on the conformational distribution of the amyloid- $\beta$ peptide based on molecular dynamics simulations. J Phys Chem B. 2007;111(49):13814-13821.

24. Fujiwara T, O’Geen H, Keles S, et al. Discovering hematopoietic mechanisms through genome-wide analysis of GATA factor chromatin occupancy. Mol cell. 2009;36(4):667-681.

25. Scally SW, Petersen J, Law SC, et al. A molecular basis for the association of the HLA-DRB1 locus, citrullination, and rheumatoid arthritis. $J$ Exp Med. 2013;210(12):2569-2582.

26. Neubauer P, Beatrix Fahnert, Hauke Lilie, et al. Protein inclusion bodies in recombinant bacteria. Inclusions in Prokaryotes. 2006;1:237-292.

27. Wong RL, Wang Q, Treviño LS, et al. Identification of secretaglobin Scgb2a1 as a target for developmental reprogramming by BPA in the rat prostate. Epigenetics. 2015;10(2):127-134

28. Scarff CA, Thalassinos K, Hilton GR, et al. Travelling wave ion mobility mass spectrometry studies of protein structure: biological significance and comparison with X-ray crystallography and nuclear magnetic resonance spectroscopy measurements. Rapid Commun Mass Spectrom. 2008;22(20):3297-3304

29. Du F, Yuan P, Wang T, et al. The Significance and Therapeutic Potential of GATA3 Expression and Mutation in Breast Cancer: A Systematic Review. Med Res Rev. 2015;35(6):1300-1315.
30. Li M, Shivender MDS, Michael AC, et al. First-in-class small molecule inhibitors of the single-strand DNA cytosine deaminase APOBEC3G. ACS chemical biology. 2012;7(3):506-517.

31. George A, Wilson WW. Predicting protein crystallization from a dilute solution property. Acta Crystallogr D Biol Crystallogr. 1994;50(4):361365 .

32. Jancarik J, SH Kim. Sparse matrix sampling: a screening method for crystallization of proteins. Journal of applied crystallography. 1991;24(4):409-411.

33. Brown JC. The deduction of energy spectra of non-thermal electrons in flares from the observed dynamic spectra of hard X-ray bursts. Solar Physics. 1971;18(3):489-502.

34. Attwood D, A Sakdinawat. X-rays and Extreme Ultraviolet Radiation: Principles and Applications. India: Cambridge university press; 2017.

35. Weik M, Ravelli RB, Kryger G, et al. Specific chemical and structural damage to proteins produced by synchrotron radiation. Proc Natl Acad Sci USA. 2000;97(2):623-628.

36. Dauter Z, Dauter M, Rajashankar KR. Novel approach to phasing proteins: derivatization by short cryo-soaking with halides. Acta Crystallogr D Biol Crystallogr. 2000;56(2):232-237.

37. Perrakis A, Morris R, Lamzin VS. Automated protein model building combined with iterative structure refinement. Nat Struct Biol. 1999;6(5):458-463.

38. Hendrickson WA. Determination of Macromolecular Structures from Anomalous Diffraction of synchrotron radiation. Science. $1991 ; 254(5028): 51-58$. 